Sharif University of Technology
Scientia Iranica
SCIENTIA
IRAN I CA

\title{
Simulation of thermally developing laminar flow in partially filled porous pipes under wall suction
}

\author{
A. Nouri-Borujerdi* and M.H. Seyyed-Hashemi \\ School of Mechanical Engineering, Sharif University of Technology, Tehran, Iran.
}

Received 28 January 2015; received in revised form 3 October 2015; accepted 4 January 2016

\author{
KEYWORDS \\ Partially filled porous \\ pipe; \\ Developing forced- \\ convection laminar \\ flow; \\ Entrance length; \\ Wall suction; \\ Numerical method.
}

\begin{abstract}
This study numerically investigates fluid flow and heat transfer enhancement of a two-dimensional developing laminar flow in an axisymmetric pipe with partially filled porous material attached to the wall. The effects of porous layer in the range of $0 \leq \delta / R<1$ and Darcy number in the range of $10^{-6} \leq \mathrm{Da} \leq 10^{-4}$ are investigated. It is found that thermal entrance length increases with porous layer thickness and is longer than a plain pipe. In addition, the dependence of Nusselt number on the thickness of the porous layer is not monotonic. A critical value of the porous layer thickness exists at which the Nusselt number value reaches the minimum. As the porous layer is thickening, more fluid is pushed towards the middle of the pipe and less fluid goes through the porous layer in comparison with the case that the porous layer is located in the center. In this case, the heat convection is reduced relative to heat conduction due to the physical contact between the solid matrix and the wall surface.
\end{abstract}

(C) 2016 Sharif University of Technology. All rights reserved.

\section{Introduction}

Heat transfer and transport phenomena in porous media are important processes in many engineering applications such as heat exchanger, electronic cooling, oil extraction, chemical catalytic reactors, and thermal insulations. It is generally known that partially filling a conduit with saturated porous media leads to the conclusion that higher heat transfer rate in a forced convection can be achieved at the expense of a reasonable pressure drop. However, the penalty of the pressure drop will be significant if the conduit is fully filled with porous media. Recently, porous media have been employed to promote heat transfer in thermal systems and this has received considerable attention [1-3]. The effects of a random porosity model on heat transfer performance of porous media are numerically demonstrated by $\mathrm{Fu}$ and Huang [4].

\footnotetext{
*. Corresponding author.

E-mail address: anouri@sharif.edu (A. Nouri-Borujerdi)
}

Deng and Martinez [5] numerically and analytically considered two-dimensional flow for partially filled porous channel under wall suction. The numerical investigation of Yucel and Guven [6] on convection cooling enhancement of heated elements in a parallelplate channel using porous inserts shows that heat transfer can be enhanced by using high thermal conductivity of porous medium. Also, the pressure drop increases rapidly along the channel with increase in the Reynolds number. Erdogan and Imrak [7] considered fully laminar flow of an incompressible viscous fluid in a uniformly porous pipe with suction and injection. They expressed the velocity filed in a series form in terms of the modified Bessel function of the first kind of order $n$. They found that flow properties depend on the suction velocity and for large values of suction velocity, the flow near the region of the suction shows a boundarylayer character. In this region, the velocity and the vortices vary sharply. Jiang and Ren [8] numerically investigated forced-convection heat transfer in porous media using a thermal non-equilibrium model. Their 
results indicated that the convection heat transfer in porous media can be predicted numerically by using the thermal non-equilibrium model with the ideal constant-wall heat-flux boundary condition. Alazmi and Vafai [9] numerically studied constant-wall heatflux boundary conditions in porous media under local thermal non-equilibrium conditions. Tzeng [10] experimentally and numerically examined the forcedconvection heat transfer in a rectangular channel filled with sintered bronze beads and periodically spaced heat blocks. The result indicated that such arrangement can be used to improve the cooling performance of heated blocks, where the blocks are used to simulate heated electronic components, by sintered metallic porous media. Aguilar-Madera [11] presented the modeling of momentum and heat transfer in parallelplate channels partially filled with a porous insert using a one-domain approach. With this methodology, they avoided specifying the boundary conditions at the fluid porous insert boundary at the cost of solving more complicated transport models. Wu et al. [12] took advantage of longitudinal vortex generator and slit to simulate heat transfer and fluid flow characteristics of composite fin numerically. Their computational results show that composite fin can improve the synergy of temperature gradient and velocity fields, and its equivalent thermal resistance is smaller and its irreversibility of heat transfer is lower. Eiamsa-ard et al. [13] and Bas and Ozceyhan [14] enhanced heat transfer in tubes by inserting twisted tapes. They stated that the optimum tradeoff between the enhancement of heat transfer and increasing of friction are obtained from a critical value between their ratio. Yang et al. [15] performed the heat transfer performance assessment for forced convection in a heated tube partially filled with a porous material in the core and attached to the wall. It was found that the local thermal non-equilibrium analysis was essential for the case of forced convection in a tube with a heated wall surface covered with a porous medium layer. On the other hand, the local thermal equilibrium analysis sufficed to capture transport phenomena for the case of forced convection in a tube with a porous medium core. In a comparatively low range of pumping power, the heat transfer performance of the tube with a porous medium core is higher than that of the tube with a wall covered with a porous medium layer. However, in a high range of pumping power, the latter performance exceeds the former. Poulikakos and Kazmierczak [16] presented a theoretical study of fully developed forced convection in a channel and a circular pipe partially filled with a porous matrix attached to the wall. Both cases of constant-wall heat flux and constant-wall temperature were studied. Of particular importance is the finding that the dependence of $\mathrm{Nu}$ on the thickness of the porous layer is not monotonic. A critical thickness exists at which the value of $\mathrm{Nu}$ reaches the minimum.
The present work numerically investigates a developing laminar forced-convection heat transfer in a pipe with partially filled porous medium. The pipe wall is under suction and at constant temperature. The effects of thermal entrance length, thickness of the porous layer, and Darcy number on the rate of heat transfer and on the pressure drop are discussed.

\section{Problem statement and formulation}

Let us assume that a laminar air flow enters a partially filled porous pipe with radius $R$ and length $L$ at uniform velocity $u_{\text {in }}$ and temperature $T_{\text {in }}$ (Figure 1 ). The wall of the pipe is reticulated at temperature $T_{w}$ and under suction. The air flow can leave the pipe both at the outlet and through the wall at atmospheric pressure. The porosity and permeability of the porous layer are assumed to be $\phi$ and $K$, respectively, with thickness $\delta$ in the range of $0<\delta<R$. The porous layer can be placed at the center or on the wall and extends inward, toward the center line.

\subsection{Mass and momentum equations}

To present the model equations in one-domain approach, a single set of unsteady transport equations including mass and momentum applicable for the entire domain comprising the clear fluid and porous layer is given as follows [17]:

$$
\begin{aligned}
& \frac{\partial u}{\partial z}+\frac{1}{r} \frac{\partial(r v)}{\partial r}=0 \\
& \frac{1}{\phi} \frac{\partial \rho u}{\partial t}+\frac{1}{\phi^{2}}\left[\frac{\partial\left(\rho u^{2}\right)}{\partial z}+\frac{1}{r} \frac{\partial(\rho r u v)}{\partial r}\right]=-\frac{d p}{d z} \\
& +\tilde{\mu}\left\{\frac{1}{r} \frac{\partial}{\partial r}\left[r \frac{\partial u}{\partial r}\right]+\frac{\partial^{2} u}{\partial z^{2}}\right\}-\frac{\mu}{K} u-\frac{\rho C_{F}}{\sqrt{K}}|u| u \\
& \frac{1}{\phi} \frac{\partial \rho v}{\partial t}+\frac{1}{\phi^{2}}\left[\frac{\partial(\rho u v)}{\partial z}+\frac{1}{r} \frac{\partial\left(\rho r v^{2}\right)}{\partial r}\right]=-\frac{d p}{d r} \\
& +\tilde{\mu}\left\{\frac{1}{r} \frac{\partial}{\partial r}\left[r \frac{\partial v}{\partial r}\right]+\frac{\partial^{2} v}{\partial z^{2}}\right\}-\frac{\mu}{K} v-\frac{\rho C_{F}}{\sqrt{K}}|v| v
\end{aligned}
$$

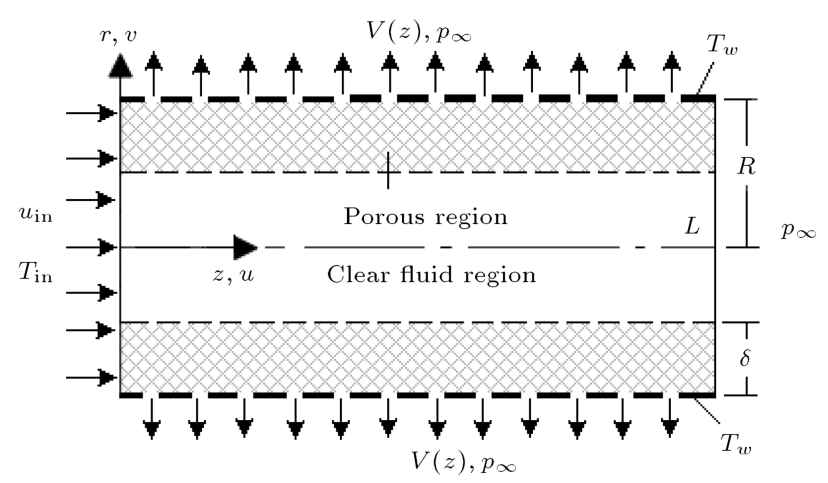

Figure 1. Schematic of the porous pipe with distribution of suction velocity through the wall. 
The corresponding momentum equations of the clear fluid are determined from Eqs. (2) and (3) when $\phi=1$ and $K \rightarrow \infty$, and the corresponding governing equations of the fluid phase in the porous layer, referred to as Brinkman-Forchheimer of Darcy's law, are obtained if $0<\phi<1$ and $K=$ finite.

The coefficient $\hat{\mu}_{f}=\mu_{f} / \phi$ is an effective liquid viscosity. Brinkman [18] set $\mu_{f}$ and $\tilde{\mu}_{f}$ equal to each other; but, in general, that is not true. Bear and Bachmat [19] suggested a detailed averaging process that led to the result: $\tilde{\mu}_{f} / \mu_{f}=1 / \phi \tau$, for an isotropic porous medium, where $\tau$ was a quantity called the tortuosity of the medium. On the other hand, straight volume averaging as presented by Ochoa-Tapia and Whitaker [20] gives $\tilde{\mu}_{f} / \mu_{f}=1 / \phi . u$ and $v$ are averaged velocity of the fluid taken with respect to a volume element of the medium (incorporating both solid and fluid materials) in $z$ - and $r$-direction, respectively. These quantities have been given various names, by different authors, such as Darcy velocity and superficial velocity. $P$ is pressure and denotes an intrinsic quantity and that Darcy's equation is not a balance of forces averaged over a representative elementary volume. Also, $C_{F}$ is a dimensionless form-drag constant and according to the results of Handley and Heggs [21], its value approaches zero when the porous Reynolds number is of order one or less. Beavers et al. [22] showed that the bounding walls could have a substantial effect on the value of $C_{F}$ and found that their data correlated fairly well with the expression $C_{F}=0.55\left(1-5.5 d / D_{e}\right)$, in which $d$ was the diameter of their sphere and $D_{e}$ was the equivalent diameter of the bed defined in terms of the height, $h$, and width, $w$, of the bed by $D_{e}=2 h w /(h+w)$. The coefficient, $K$, is called the specific or intrinsic permeability and is independent of the nature of the fluid, but it depends on the geometry of the medium. It is in general a second-order tensor. For the case of an isotropic medium, it is a scalar quantity and its value for natural materials varies widely with dimension. If $K$ is determined by the geometry of the medium, then, clearly, it is possible to calculate $K$ in terms of the geometrical parameters. For example, in the case of beds of particles or fibbers, one can introduce an effective average particle or fiber diameter $d_{\text {peff }}$. The hydraulic radius theory of Carman-Kozey leads to the relationship:

$$
K=\frac{d_{\mathrm{peff}}^{2} \phi^{3}}{150(1-\phi)^{2}},
$$

where:

$$
d_{\mathrm{peff}}=\frac{\int_{0}^{\infty} d_{p}^{3} f\left(d_{p}\right) d\left(d_{p}\right)}{\int_{0}^{\infty} d_{p}^{2} f\left(d_{p}\right) d\left(d_{p}\right)}
$$

$f\left(d_{p}\right)$ is the density function of the distribution of diameter $d_{p}$. The constant 150 in Eq. (4) was obtained by seeking the best fit with experimental results.

\subsection{Energy equation}

We start with a simple situation in which the medium is isotropic and where radiation effect, viscous dissipation, and the work done by pressure changes are absent. Here, we assume that heat conduction in the fluid and solid phases takes place in parallel so that there is no net heat transfer from one phase to the other. Usually, it is a good approximation to assume that the solid and fluid phases are in thermal equilibrium; but there are situations, such as highly transient problem and some steady-state problems, in which this is not so [23].

$$
\begin{aligned}
\left(\rho C_{p}\right)_{\operatorname{stag}} & \frac{\partial T}{\partial t}+\rho_{f} C_{p f}\left[\frac{\partial u T}{\partial z}+\frac{1}{r} \frac{\partial r v T}{\partial r}\right] \\
= & {\left[k_{\text {stag }}+\phi k_{\text {dis }}\right]\left\{\frac{1}{r} \frac{\partial}{\partial r}\left[r \frac{\partial T}{\partial r}\right]+\frac{\partial^{2} T}{\partial z^{2}}\right\} }
\end{aligned}
$$

The corresponding energy equation of the clear fluid is determined from Eq. (6), where the porosity is $\phi=1$ and the corresponding energy equation of the porous layer is obtained if $0<\phi<1$. Meanwhile, $k_{\text {stag }}=$ $\phi k_{f}+(1-\phi) k_{s}$ and $\left(\rho C_{p}\right)_{\text {stag }}=\phi \rho_{f} C_{p f}+(1-\phi) \rho_{s} C_{p s}$. The effective thermal conductivity of the fluid consists of the stagnant and dispersion components as $k_{f \text { eff }}=$ $k_{f}+k_{\text {dis }}$, which is expressed by empirical correlation of Wakao and Kaguei [24].

$$
k_{f \mathrm{eff}}=k_{f}\left[1+0.1 \frac{\operatorname{Re}_{p} \operatorname{Pr}}{\phi}\right] \text {. }
$$

Also, Calmidi and Mahajan [25,26] proposed the following correlations:

$$
\begin{aligned}
& k_{\text {stag }}=\phi k_{f}+0.19 k_{s}(1-\phi)^{0.763}, \\
& k_{\text {dis }}=0.06 \frac{\rho_{f} C_{p f} u_{D} \sqrt{K}}{\phi}
\end{aligned}
$$

in which $u_{D}$ is Darcian velocity (uniform inlet velocity). The Nusselt number is:

$$
\mathrm{Nu}=\frac{\dot{q}_{w}^{\prime \prime} D}{\left(T_{b}-T_{w}\right)\left(k_{\mathrm{stag}}+\phi k_{\mathrm{dis}}\right)},
$$

where, $T_{b}$ is the bulk or mean temperature of the stream across the pipe and is calculated by:

$$
T_{b}=\frac{1}{A u_{\mathrm{in}}} \int_{A} u T d A .
$$




\section{Boundary conditions}

At the interface between the wall and the porous layer or between a porous layer and a clear fluid, we can impose continuity of the normal component of the heat flux and continuity of the temperature because of the assumption of local thermal equilibrium.

The corresponding boundary conditions of the above governing equations are specified as follows:

$$
\begin{aligned}
& u(r, 0)=u_{\mathrm{in}}, \quad v_{\mathrm{in}}(r, 0)=0, \quad T_{f}(r, 0)=T_{\mathrm{in}}, \\
& \frac{\partial u(r, L)}{\partial z}=0, \quad \frac{\partial v(r, L)}{\partial z}=0 \\
& \frac{\partial T_{f}(r, L)}{\partial z}=0, \quad \frac{\partial T_{s}(r, L)}{\partial z}=0 \\
& P(r, L)=P_{\infty}, \quad \frac{\partial T_{f}(0, z)}{\partial r}=0 \\
& \frac{\partial u(0, z)}{\partial r}=0, \quad p(R, z)=p_{\mathrm{atm}}, \\
& v(0, z)=0, \quad T_{f}(R, z)=T_{s}(R, z)=T_{w} .
\end{aligned}
$$

Indices $s$ and $f$ refer to solid and fluid, respectively. Since the outside of the pipe is exposed to the atmospheric pressure and is constant along the boundary for all values of $z, \partial p / \partial z=0$ and, hence, $u=0$ for all $z$. Therefore, based on the continuity equation, $\partial u / \partial z=0$ at $r=R$. We conclude that:

$$
\frac{\partial v(R, z)}{\partial r}=0 \text {. }
$$

\section{Numerical method with one-domain approach}

To determine the flow variables in the clear fluid and in the porous layer, Eqs. (1)-(5) have been solved numerically in one-domain approach with an interface zone across which the porosity and permeability are continuous. In the one-domain approach, the interface can be either a continuous transition zone across which the physical variables encounter possibly strong but nevertheless continuous variations (e.g., Goyeau et al. [27] and Valdes-Parada et al. [28]) or a discontinuous interface where the physical variables are possibly discontinuous (e.g., Silva and de Lemos [29]). Jamet and Goyeau [30] show that the discontinuous onedomain approach and the two-domain approach are mathematically strictly equivalent, provided that the discontinuous one-domain approach is in the sense of distributions. On the other hand, the experimental results of Basu and Khalili [31] show that a one-domain approach can provide good predictions of interfacial flow. In this regard, Arquiset et al. [32] used a harmonic mean formulation of the permeability and Poulikakos [33] also used a hyperbolic tangent function to handle the abrupt change in governing parameters.

In this work, a finite-volume method in onedomain approach is used to discretize the governing equations based on a staggered grid with uniform grid spacing in $r$-and $z$-direction. We also used a hyperbolic tangent function to handle the abrupt change in the porosity and permeability in the interface zone. The fluid-porous interface is supposed to be located at the center of the cell interface. Scalars variables are stored at the center of the mesh cells and variables of the vectors are stored at the mesh faces. The second-order upwind differencing scheme is used for convection terms and the central differencing scheme is used for diffusion terms. The pressure and velocity linked in the momentum equations are solved on the staggered grid arrangement. Subsequently, the set of the discretized linear algebraic equations for $u, v$, and $p$ are solved using successive line-by-line relaxation by tri-diagonal matrix algorithm. Either of the algebraic equations includes only three non-zero coefficients. The convergence criterion for solution of equations is also established by local and global mass and energy conservations in which their corresponding residuals are set equal to $10^{-6}$.

\section{Results and discussion}

It is assumed that air flow enters a partially filled porous pipe with radius $R=2 \mathrm{~cm}$ and length $L=$ $50 \mathrm{~cm}$ at uniform inlet velocity $u_{\text {in }}=0.805 \mathrm{~m} / \mathrm{s}$ and temperature $T_{\mathrm{in}}=25^{\circ} \mathrm{C}$ (see Figure 1$)$. The air flow exits through both the outlet and the permeable wall of the pipe at temperature $T_{w}=35^{\circ} \mathrm{C}$ and atmospheric pressure. The porous layer is placed in the center or attached to the pipe wall and extends inward, toward the centerline. The porous material is made of a commercial aluminum with physical properties of $\rho=$ $2770 \mathrm{~kg} / \mathrm{m}^{3}, C_{p}=875 \mathrm{~J} / \mathrm{kg} .{ }^{\circ} \mathrm{K}, k=177 \mathrm{~W} / \mathrm{m} . \mathrm{K}$, and porosity of $\phi=0.7$.

Model tests: In order to validate the numerical results and to show that the results are independent of the grid size, a number of grids with different sizes are used. To compare the present results with the previously published results in the open literature, Figures 2 and 3 are plotted for the case in which the porous layer is placed in the center of the pipe without wall suction. These figures respectively show the mesh refinement and axial velocity at the outlet cross section of a pipe with radius $R=3.2 \mathrm{~cm}$ and length $L=50 \mathrm{~cm}$ for four grid points $8 \times 13,17 \times 26,33 \times 51$, and $65 \times 101$. It is found that the velocity will be independent grid 


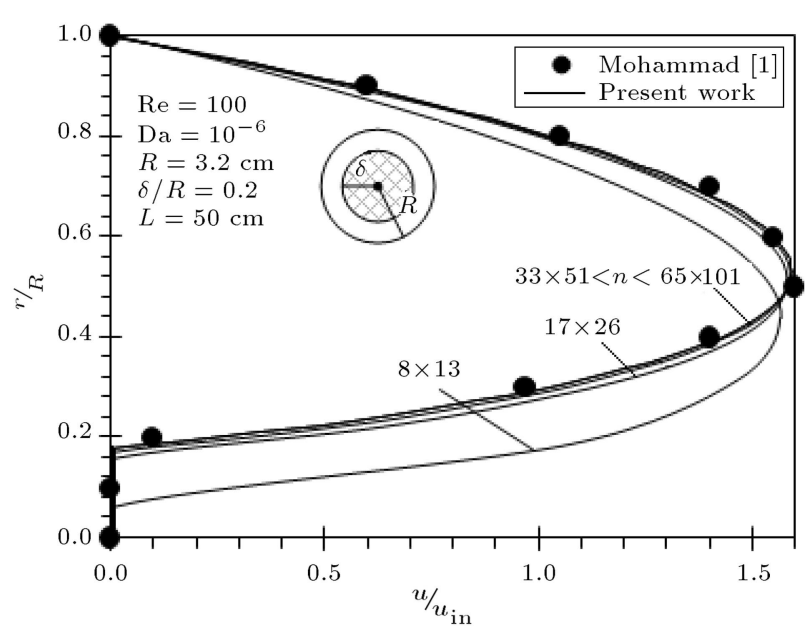

Figure 2. Mesh refinement for the axial velocity at the outlet cross section of the pipe and the results of Mohammad [1] without wall suction.

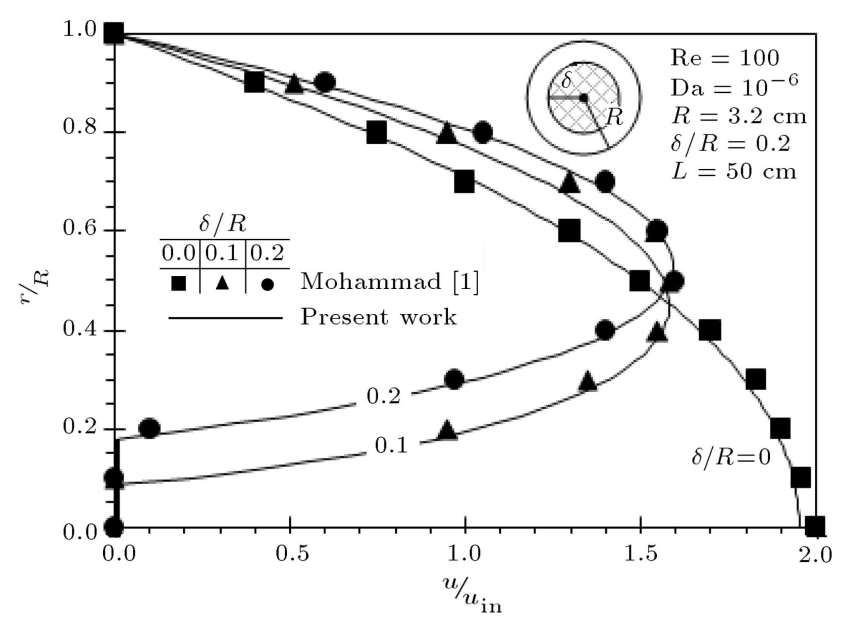

Figure 3. Axial velocity for different thicknesses of the porous layer located in the pipe center and the results of Mohammad [1] without wall suction.

size when the grid points are equal to or greater than $65 \times 101$. In this figure, the data of Mohammad [1] is also given for comparison. The other cases where the porous layer is attached to the wall have been plotted in Figures 3-9.

Model results: Figure 3 depicts three axial velocity profiles at the outlet cross section of the pipe $(z=L)$ for two different porous layers $(\delta / R=0.1,0.2)$ located in the center of the pipe. The third one $(\delta / R=0)$ corresponds to the empty pipe or no porous layer. The data of Mohammad [1] is also shown for three layers, i.e. $\delta / R=0,0.1$ and 0.2 . The comparison shows a good agreement between these three cases. It is worth noting that the velocity profile in the porous layers for $\delta / R=0.1$, and 0.2 is Darcy flow and for the clear pipe $(\delta / R=0)$ is Poiseuille flow with maximum speed at the center of the pipe.

Figure 4 represents the fully developed axial ve-

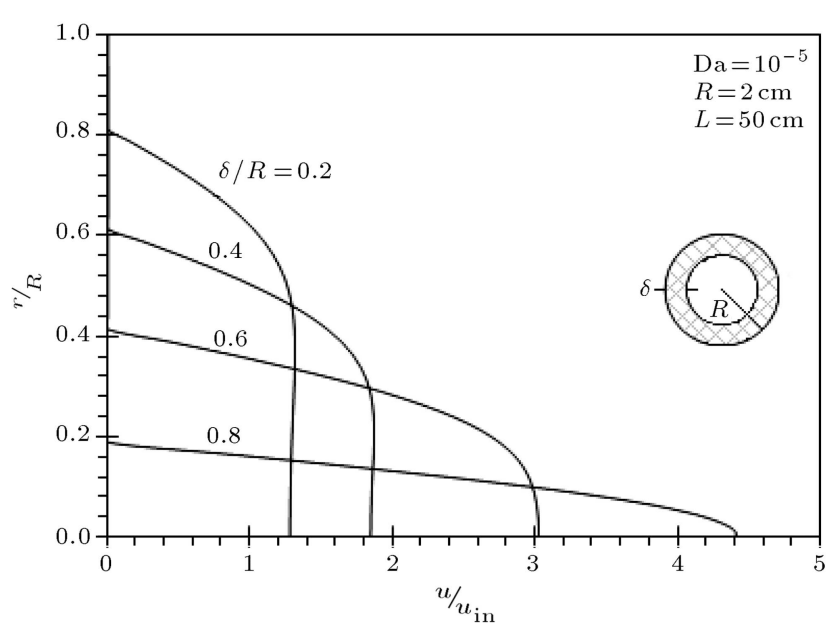

Figure 4. Profiles of the developed axial velocity for different thicknesses of porous layer in the vicinity of the wall with suction.

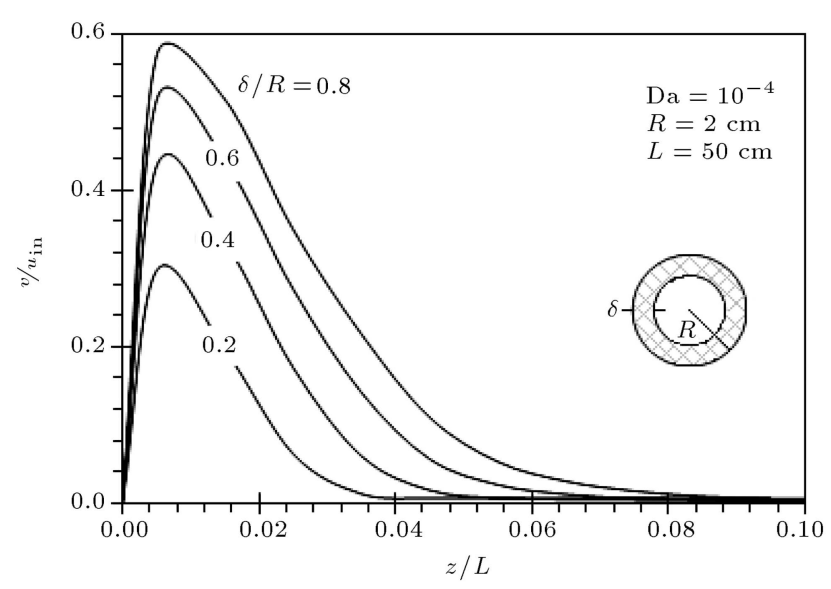

Figure 5. Distribution of suction velocity through the wall for different thicknesses of the porous layer.

locity profiles for four different porous layer thicknesses $(\delta / R=0.2,0.4,0.6,0.8)$ at the outlet cross section of the pipe with wall suction. In this case, the porous layer is attached to the wall. As the porous layer is thickening, more fluid is pushed towards the middle of the pipe and the maximum speed occurs at the center. In other words, less mass flow rate of air goes through the porous layer in comparison with the case that the porous layer is located in the central region.

Figure 5 depicts distribution of suction velocity through the wall for four different thicknesses of porous layer in the vicinity of the wall $(\delta / R=0.2,0.4,0.6$ and $0.8)$. The coordinates axes have been dimensionless by the inlet velocity and the length of the pipe. The discharge velocity increases sharply near the intake pipe; then, it gradually decreases and eventually reaches zero. Furthermore, the thicker layer makes the emptying rate increase. It can be deduced from Darcy law that emptying rate is proportional to the pressure gradient.

Figure 6 represents the pressure distribution in 


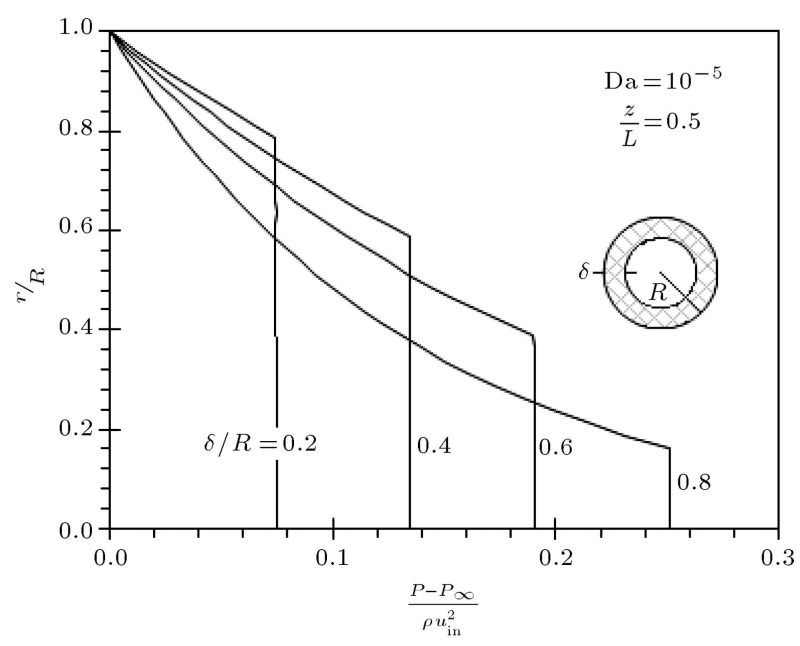

Figure 6. Distribution of radial pressure in the middle length of the pipe for different thicknesses of porous layer attached to the wall with suction.

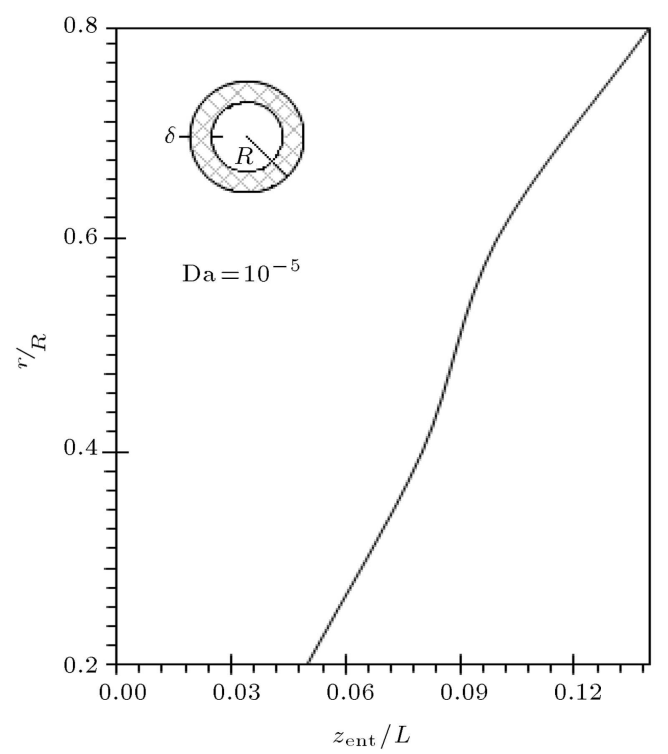

Figure 7. Thermal entrance length versus the porous layer thickness with wall suction.

the $r$-direction and in the middle length of the pipe for different thicknesses of porous layer attached to the wall with suction. Each curve consists of two parts, a vertical part that belongs to the clear fluid region with constant pressure and the other part belongs to the porous layer with a pressure reduction. In the porous layer, the pressure starts from the atmospheric pressure at the wall and reaches the pressure of air flow at the interface between two regions. For instance, when $\delta / R=0.6$, air pressure in the middle of the pipe is $\left(p-p_{\infty}\right) / \rho u_{\text {in }}^{2}=0.19$; then, it gradually decreases across the porous layer and reaches the atmospheric pressure at the wall.

Figure 7 exhibits the thermal entrance length against the porous layer thickness. It is found that the entrance length increases somehow linearly with

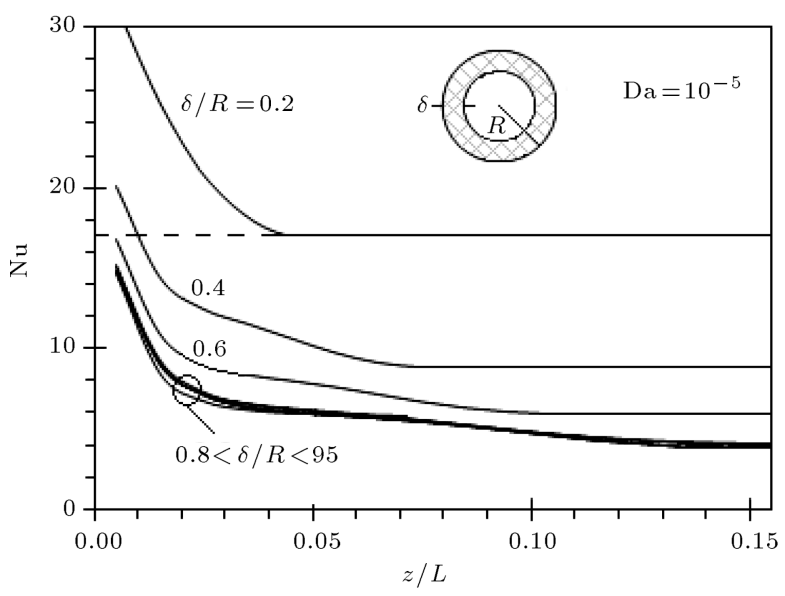

Figure 8. Developing local Nusselt number along the pipe for different porous layer thicknesses attached to the wall with suction.

porous layer thickness and is approximated by function of $z_{\text {ent }} / L=0.135 \delta / R$. Thickening of the porous layer pushes the fluid towards the middle of the pipe and more fluid goes through the clear fluid region. In other words, the corresponding Reynolds number increases and thermal entrance length and, consequently, heat transfer coefficient and pressure drop increase as well.

Figure 8 illustrates the developing local Nusselt number along the pipe for four different porous layer thicknesses $(0.2,0.4,0.6,0.8)$ and Darcy number of $\mathrm{Da}=10^{-5}$. Each curve, after passing a distance from the inlet pipe, reaches a constant value. This distance corresponds to the thermal entrance length and depends on the thickness of the porous layer.

Figure 9 illustrates the fully developed Nusselt number versus the porous layer thickness without consideration of convective terms in momentum equations as well as without wall suction. A surprising finding is that dependence of the Nusselt number on the thickness of the porous layer is not monotonic. That is, there exists a critical value of the porous layer thickness at which the value of the Nusselt number reaches the minimum. The physical interpretation is that thickening of the porous layer pushes the fluid towards the middle of the pipe and less mass flow rate goes through the porous layer. In this event, the rate of convective heat transfer between the wall surface and the fluid saturated porous layer is reduced in comparison with the conduction part due to the contact between the solid matrix and the wall surface. The figure also includes the data of Poulikakos and Kazmierczak [16] for comparison.

\section{Conclusion}

This study has numerically considered a developing laminar forced-convection heat transfer in a partially filled porous pipe with wall suction for the range of 


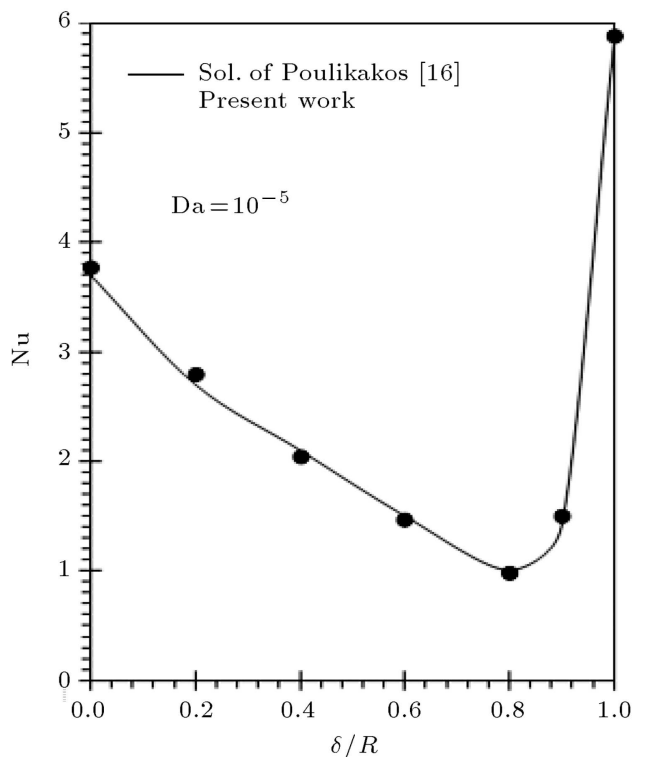

Figure 9. Fully developed Nusselt number versus the porous layer thickness and in comparison with the results of Popukakous [16] without wall suction.

Darcy number, $10^{-6} \leq \mathrm{Da} \leq 10^{-4}$, and porous layer thickness, $0 \leq \delta / R<1$. It is found that thermal entrance length increases with porous layer thickness and is longer than that of the pipe with no porous layer. Further, the local Nusselt numbers approach the developed value after a distance from the inlet of the pipe and depend on the porous layer thickness. Due to the resistance of the porous matrix to the fluid flow, less mass flow rate goes through the porous layer in comparison with the case that the porous layer is located in the center. In this case, the rate of convection heat transfer between the wall and the fluid saturated porous region is reduced relative to the heat conduction between them.

\section{Nomenclature}

$A \quad$ Surface area

$C_{p} \quad$ Specific heat

$C_{F} \quad$ Inertial coefficient

$D \quad$ Pipe diameter

Da Darcy number $K / D^{2}$

$d_{p} \quad$ Particle diameter

$h \quad$ Heat transfer coefficient

$k \quad$ Thermal conductivity

$K \quad$ Permeability

$L \quad$ Length

$\mathrm{Nu} \quad$ Nusselt number, $h d / k$

$\dot{q}^{\prime \prime} \quad$ Heat flux

$p \quad$ Pressure

$\begin{array}{ll}\operatorname{Pr} & \text { Prantel number } \\ r & \text { Radial coordinate } \\ R & \text { Pipe radius } \\ \operatorname{Re} & \text { Reynolds number, } v D / \nu \\ t & \text { Time } \\ T & \text { Temperature } \\ u & \text { Axial velocity } \\ v & \text { Radial coordinate } \\ z & \text { Axial coordinate }\end{array}$

\section{Greek letters}

$\alpha \quad$ Thermal diffusivity, $k /\left(\rho C_{p}\right)$

$\delta \quad$ Porous wall or porous layer thickness

$\mu \quad$ Dynamic viscosity

$\nu \quad$ Kinematic viscosity

$\rho \quad$ Density

$\phi \quad$ Porosity

\section{Subscripts}

$\begin{array}{ll}\text { atm } & \text { Atmosphere } \\ \text { dis } & \text { Dispersion } \\ \text { eff } & \text { Effective } \\ f & \text { Fluid } \\ \text { in } & \text { Inlet conditions } \\ p & \text { Porous, particle } \\ s & \text { Solid } \\ \text { stag } & \text { Stagnation } \\ w & \text { Wall }\end{array}$

\section{References}

1. Mohamad, A.A. "Heat transfer enhancement in heat exchangers fitted with porous media Part I: constant wall temperature", Int. J. Thermal Science, 42, pp. 385-395 (2003).

2. Pavel, B.I. and Mohamad, A.A. "An experimental and numerical study of heat transfer gas heat exchanger fitted with porous media", Int. J. Heat and Mass transfer, 47, pp. 4939-4952 (2004).

3. Huang, P.C. and Vafai, K. "Analysis of forced convection enhancement in a channel using porous blacks", AIAA J. Thermophysic, Heat Transfer, 8, pp. 563-573 (1994).

4. Fu, W.S. and Huang, H.C. "Effects of a random porosity model on heat transfer performance of porous media", Int. J. Heat Mass Transfer, 42, pp. 13-25 (1999).

5. Deng, C. and Martinez, D.M. "Viscous flow in a channel partially filled with a porous medium and with wall suction", Engineering Science, 60(2), pp. 329-336 (2005). 
6. Yucel, N. and Guven, R.T. "Forced-convection cooling enhancement of heated elements in a parallel-plate channels using porous inserts", Heat Transfer Part A, 51, pp. 293-312 (2007).

7. Erdogan, M.E. and Imrak, C.E. "On the flow in a uniformly porous pipe", Int. J. of Non-Linear Mechanics, 43, pp. 292- 301 (2008).

8. Jiang, P.X. and Ren, Z.P. "Numerical investigation of forced convection heat transfer in porous media using a thermal non-equilibrium model", Int. J. Heat Fluid Flow, 22(1), pp. 102-110 (2001).

9. Alazmi, B. and Vafai, K. "Constant wall heat flux boundary conditions in porous media under local thermal non-equilibrium conditions", Int. J. Heat Mass Transfer, 45, pp. 3071-3087 (2002).

10. Tzeng, S.C. "Convective heat transfer in a rectangular channel filled with sintered bronze bead and periodically spaced heated blocks", Trans. ASME J. Heat Transfer, 128, pp. 453-464 (2006).

11. Aguilar-Madera, C.G., Valdés-Parada, F.J., Goyeau, B. and Ochoa-Tapia, J.A. "Convective heat transfer in a channel partially filled with a porous medium", International Journal of Thermal Sciences, 50, pp. 1355-1368 (2011).

12. Wu, X., Zhang, W., Gou, Q., Luo, Z. and Lu, Y. "Numerical simulation of heat transfer and fluid flow characteristics of composite fin", International Journal of Heat and Mass Transfer, 75, pp. 414-424 (2014).

13. Eiamsa-ard, S., Somkleang, P., Nuntadusit, C. and Thianpong, C. "Heat transfer enhancement in tube by inserting uniform/non-uniform twisted-tapes with alternate axes: Effect of rotated-axis length", Applied Thermal Engineering, 54(1), pp. 289-309 (2013).

14. Bas, H. and Ozceyhan, V. "Heat transfer enhancement in a tube with twisted tape inserts placed separately from the tube wall", Experimental Thermal and Fluid Science, 41, pp. 51-58 (2012).

15. Yang, C., Nakayama, A. and Liu, W. "Heat transfer performance assessment for forced convection in a tube partially filled with a porous medium", Int. Journal of Thermal Sciences, 54, pp. 98-108 (2012).

16. Poulikakos, D. and Kazmierczak, M. "Forced convection in a duct partially filled with a porous material", ASME Trans., J. of Heat Transfer, 109, pp. 653-662 (1987).

17. Nield, D.A. and Bejan, A., Convection in Porous Media, Chap 2, 4th Edn., Springer, New York (2013).

18. Brinkman, H.C. "On the permeability of media consisting of closely packed porous particles", Appl. Sci. Res. A., pp. 81-86 (1947b).

19. Bear, J. and Bachmat, Y., Introduction to Modeling of Transport Phenomena in Porous Media, Kluwer Academic Publishers, Dordrecht, The Netherlands, 177 (1990).

20. Ochoa-Tapia, J.A. and Whitaker, S. "Momentum transfer at the boundary between a porous medium and a homogeneous fluid- I: Theoretical development", Int. J. Heat and Mass Transfer, 38, pp. 2635-2646 (1995a).

21. Handley, D. and Heggs, P.J. "Momentum and heat transfer mechanisms in regular shaped packings", Trans. Inst. Chem. Engrs., 46, pp. T251-T264 (1968).

22. Beavers, G.S., Sparrow, E.M. and Rodenz, D.E. "Influence of bed size on the flow characteristics and porosity of randomly packed beds of spheres", J. Appl. Mech., 40, pp. 655-660 [1.5.2] (1973).

23. Nield, D.A. "Effects of local thermal nonequilibrium in steady convective processes in a saturated porous medium: forced convection in a channel", J. Porous Media, 1, pp. 181-186 [2.2.3, 4.10] (1998a).

24. Wakao, N. and Kaguei, S., Heat and Mass Transfer in Packed Beds, Gordon and Breach Science Publishers, p. 364, New York, NY (1982).

25. Calmidi, V.V. and Mahajan, R.L. "The effective thermal conductivity of high porosity fibrous metal foams", ASME Trans. J. Heat Transfer, 121, 466e 471 (1999).

26. Calmidi, V.V. and Mahajan, R.L. "Forced convection in high porosity metal foams", ASME Trans. J. Heat Transfer, 122, 557e565 (2000).

27. Goyeau, B., Lhuillier, D., Gobin, D. and Velarde, M.G. "Momentum transport at a fluid-porous interface", Int. J. Heat Mass Transfer, 46, pp. 4071-4081 (2003).

28. Valdes-Parada, F.J., Goyeau, B. and Ochoa-Tapia, J.A. "Diffusive mass transfer between a microporous medium and an homogeneous fluid: Jump boundary conditions", Chem. Eng. Sci., 61(5), pp. 1692-1704 (2006).

29. Silva, R.A. and de Lemos, M.J.S. "Numerical analysis of the stress jump interface condition for laminar flow over a porous layer", Numerical Heat Transfer, Part A, 43(6), pp. 603-617 (2003a).

30. Jamet, D. and Goyeau, C.M. "On the equivalence of the discontinuous one- and two-domain approachs for the modeling of transport phenomena at a fluid/porous interface", Transport Porous Media, 78, pp. 403-418 (2009).

31. Basu, A.J. and Khalili, A. "Computation of flow through a fluid-sediment interface in a benthic chamber", Physics of Fluids, 11(6), pp. 1395-1405 (1999).

32. Arquiset, E., Caltagirone, J.P. and Langlais, C. "Natural convection in cavities partially filled with permeable porous materials", In Proceedings of the 8th Int. Heat Transfer Conference, 2, p. 2653 (1986). 
33. Poulikakos, D.M. "Thermal instability in a horizontal fluid layer superposed on a heat generating porous bed", Numerical Heat Transfer, 12(83), pp. 83-99 (1987).

\section{Biographies}

Ali Nouri-Borujerdi is Professor of Mechanical Engineering at Sharif University of Technology, Tehran, Iran, where he teaches undergraduate- and graduatelevel courses in the Thermal/Fluids Sciences Group of Mechanical Engineering Department. His teaching focuses on heat transfer, computational fluid dynamics, and two-phase flows, including boiling and condensation. His current research programs include numerical simulation of two-phase flow, compressible turbulent flows, and porous media. Professor Nouri has published more than 140 articles in international journals and conferences.

Mohammad-Hossein Seyyed-Hashemi received his BS degree in Mechanical Engineering from Iran University of Sciences and Technology. He received MS degree in Thermal Fluid Sciences from Mechanical Engineering Department at Sharif University of Technology, Tehran, Iran. 